\title{
RADIO FREQUENCY BASED WATER LEVEL MONITOR AND CONTROLLER FOR RESIDENTIAL APPLICATIONS
}

\author{
D. B. N. Nnadi ${ }^{1}{ }^{*}$, S. E. Oti ${ }^{2}$ and P. C. Ezika ${ }^{3}$ \\ 1, 2, 3 Department of Electrical EngineERING, University of Nigeria, NSUKKa, ENUGU STATE. NigERIA \\ E-mail address: ${ }^{1}$ damian.nnadi@unn.edu.ng,2stephen.oti@unn.edu.ng,3naijaboy4live@gmail.com
}

\begin{abstract}
This paper elucidates a radio frequency (RF) based transmission and reception system used to remotely monitor and control the water Level of an overhead tank placed up to 100 meters away from the pump and controller. It uses two Radio Frequency transceivers along with a controller each installed at the overhead tank and the sump (reservoir). It is completely automated with the help of a PIC 16F877A micro controller which receives signals from the transmitter, interprets and effectively communicates the status of the water overhead tank and water pump mode to the user or operator via an integrated LCD display. Similarly, the control unit of the prototype performs automatic switching control of on and off on a single phase centrifugal water pump, 220volts, $0.5 \mathrm{hp}$ motor via a motor driver circuit (relay). It also incorporates a buzzer that beeps briefly when water level hits $100 \%$, thus causing the pump to be switched off but when water level drops below 25\%, the pump is then switched on.
\end{abstract}

Key words: radio frequency, PIC microcontroller, encoder, decoder, water pump, residential.

\section{INTRODUCTION}

Sustainability and management of available water resource in many residential homes and industries are now dominant issues especially in this part of the world where three to four or more floors are rampart as residential houses. The problem of carrying water to the upper floors of such residential buildings arises since tap water is not always available and may not have such force to move up to such height. Consequently, due to the distance and nature of house hold and industrial water overhead tanks, it is very difficult, time consuming and even sometimes it is impossible for water users to accurately monitor and control the water level of a standard conventional overhead water tank system. Most times the only way to know when an overhead tank is full is when it starts overflowing which encourages water wastage since there is no efficient means of knowing when the water is low. Therefore, proper monitoring is needed to ensure that water sustainability is actually maintained, with disbursement linked to sensing and automation. As a developing nation, Nigeria lack adequate and integrated water management. In order to ameliorate such problem, then this research work was conceived and given birth to.
There are several monitoring systems integrated with water level detection which have been in literature. First among them is magnetic and mechanical float method. The principle behind magnetic and mechanical float level sensors involves the opening or closing of a mechanical switch, either through direct contact with the switch, or magnetic operation of a reed. With magnetically actuated float sensors, switching occurs when a permanent magnet sealed inside a float rises or falls to the actuation level [1]. Secondly, in the Ultrasonic method, ultrasonic level sensors are used for non-contact level sensing of highly viscous liquids, as well as bulk solids. They are also widely used in water treatment applications for pump control and open channel flow measurement. The sensors emit high frequency ( $20 \mathrm{kHz}$ to $200 \mathrm{kHz}$ ) acoustic waves that are reflected back to and detected by the emitting transducer [2-4]. In addition, optical interface method is another method; in this method optical sensors are used for point level sensing of sediments, liquids with suspended solids, and liquid-liquid interfaces. These sensors sense the decrease or change in transmission of infrared light emitted from an infrared diode (LED). With the proper choice of construction materials and mounting location, these sensors can be used with 
aqueous, organic, and corrosive liquids [5]. A common application of economical infrared-based optical interface point level sensors is used in detecting the sludge/water interface in settling ponds. By using pulse modulation techniques and a high power infrared diode, one can eliminate interference from ambient light, operate the LED at a higher gain, and lessen the effects of build-up on the probe. According to K. Govinda et al [6], their research work proposed using some kind of LED light for the indication of water level. By touching different water levels through water level sensor, LED should be indicated as on/off.

In this paper a radio frequency (RF) based transmission and reception system is used to remotely monitor and control the water Level of an overhead tank. Based on the findings of Khaled Reza el al [7], they introduced the concept of water level monitoring and management within the context of electrical conductivity of the water. Hence, when the sensor touches water, nozzles and connecting rod get electric connection using water conductivity [8]. The level of water in the overhead tank is being monitored and at certain level of water $25 \%$ empty, the system automatically switches the pumping machine on to pump water into the overhead tank and then switches off when the water is $100 \%$ full. The control section of this research work is implemented using PIC Microcontroller. Using this system, one can remotely monitor and at the same time control the water level of an overhead tank that is placed up to 100 meters high from base.

System Block Diagram:

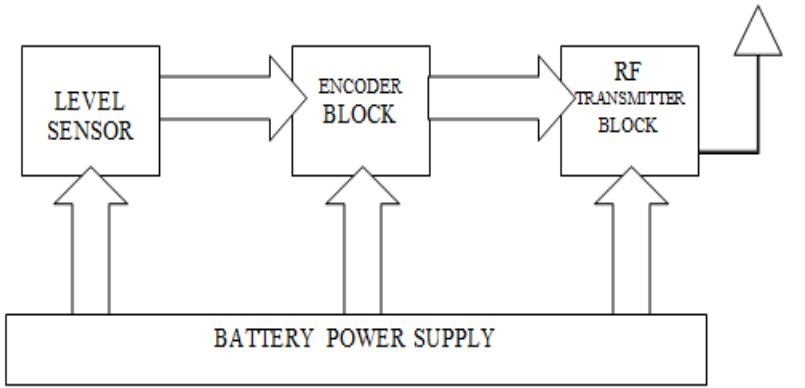

Figure 1: Sensor/Transmitter block

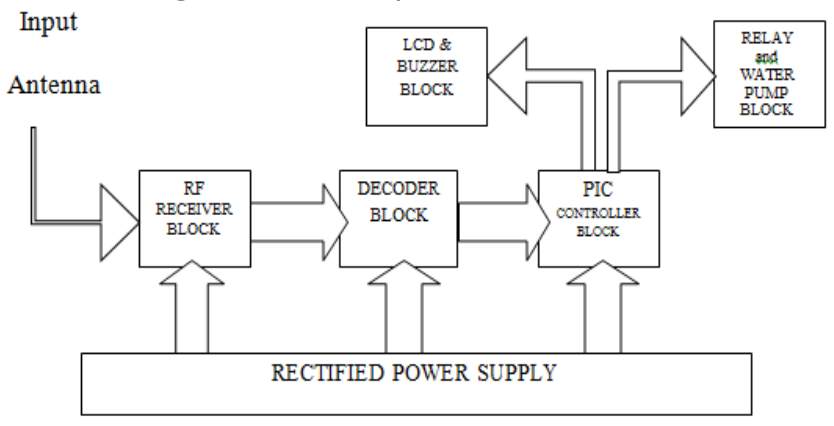

Figure 2: Receiver/Controller block
Ejiofor and Oladipo [9] did a similar work, and according to their research work when water touches the copper sensor positioned at a particular level in the tank, voltage is transferred to the copper which in turn is transferred to the comparator circuit for further processing. Which means the copper rod is inside the water which may contaminate the water with time due to electrolysis, but in this work pure stainless probes are used and placed at different levels of the tank such that whenever water touches the probe a signal is sent to the transmitter. Also, the paper did not specify the range the wireless can cover but in this prototype, it covers range of 100 meters away from the base. The prototype being a local product is lower in price when compared with its contemporary in the market. Secondly, in the event of fault its trouble shooting and maintenance are always easing.

\section{RF BASED WATER LEVEL INDICATOR / CONTROLLER}

The system features an RF transmitter-receiver pair that saves running wires from the roof to ground. The water level sensing section senses the level of water in the overhead tank and sends signal to the receiver section which is at the base of the building. The receiver section is connected to the controlling section, which process the received information and produces visual, sound indications and controls the operation of motor whenever required. The sub-circuits that make up the whole work includes: Power Supply unit, encoder / transmitter sub-circuit, decoder / receiver sub-circuit, control system sub-circuit.

The power supply unit provides the needed stable power supply to the system, the sensed water level is streamed wirelessly through an RF transmitter in the encoder /transmitter sub-circuit mounted on the overhead thank. This RF data sent from the transmitter is received by the receiver sub-circuit placed remotely and decoded. The decoded data is collected by the control system sub-circuit for processing and indicates the water level on an LCD as well as performing automatic switching control on a single phase 220volts $0.5 \mathrm{hp}$ water pump. It also incorporates a buzzer that beeps briefly when water level hits $100 \%$ and when water level drops below $25 \%$.

\section{MAJOR COMPONENT DESCRIPTIONS:}

The major circuit components and modules used in the implementation of this system are as follows: Single Phase Centrifugal Water Pump, PIC Microcontroller, Liquid Crystal Display (LCD), RF Module HT12E 
Encoder, and HT12D Decoder. Figures 1 and 2 show all the sections of this prototype.

\subsection{Centrifugal Electric Water Pump:}

For this work a low powered centrifugal pump is used, the pump is synchronized between a miniature water reservoir and a miniature overhead tank. The designed and constructed water level indicator controller makes the decision on when to activate or deactivate the water pump automatically.

The water pump is controlled by connecting it to an output pin of microcontroller via a motor driver circuit. When Microcontroller sends either in a positive signal $(+5 \mathrm{v})$ or a ground signal $(0 \mathrm{v})$ to the motor driver circuit, then the water pump becomes on or off respectively

Table 1: Water Pump Specifications

\begin{tabular}{|c|c|c|c|}
\hline \multicolumn{4}{|l|}{$\overline{D A B}$} \\
\hline \multicolumn{2}{|l|}{ QB60 } & N.09.09 & $\mathrm{CE}$ \\
\hline \multicolumn{2}{|c|}{ Q $0.2-2.2 \mathrm{~m}^{3} / \mathrm{h}$} & H $32-12 \mathrm{~m}$ & $0.5 \mathrm{HP}$ \\
\hline \multicolumn{2}{|c|}{$\mathrm{H} \max 35 \mathrm{~m}$} & $\mathrm{H} \min 10 \mathrm{~m}$ & $0.372 \mathrm{KW}$ \\
\hline \multicolumn{2}{|c|}{$220-240 \mathrm{~V} \sim$} & $2.3 \mathrm{~A}$ & $50 \mathrm{~Hz}$ \\
\hline $\begin{array}{l}2800 \\
\mathrm{r} / \mathrm{min}\end{array}$ & IP44 & I.CL.F & $8 \mu \mathrm{F} 450 \mathrm{~V} \sim$ \\
\hline
\end{tabular}

Table 1 is the name-plate copy of the actual DAB water pump used for this work.

\section{RF TRANSCEIVER}

$\mathrm{RF}$ modules are mostly used in medium and low volume products for consumer applications such as garage door openers, wireless alarm system, industrial remote controls, smart sensor applications, and wireless home automation systems. They are sometimes used to replace older infrared communication designs as they have the advantage of not requiring line-of-sight operation [10].

Basically a transceiver is a module which has a transmitter and a receiver. Here we use a R.F transceiver which transmits and receives R.F signal. The frequency range of the signal is $433 \mathrm{MHz}$. The module modulates the data using a carrier signal of frequency $433 \mathrm{MHz}$ and transmits through an antenna. On the other hand it demodulates the received signal. The modulation which takes place here is amplitude shift keying. The module requires an external antenna connected to it to transmit the signal. The length of the antenna is $1 / 4$ th of the wavelength of the signal. And wavelength $(\chi)$ is calculated from

$$
\mathrm{C}=\mathrm{f} \lambda
$$

Where $\mathrm{C}$ is the speed of light, $\mathrm{f}$ is the frequency of module and $\lambda$ is the wavelength of the signal. For the $433 \mathrm{MHz}$ module it is around $17 \mathrm{~cm}$. These transceivers are placed at the sump and the tank for the purpose of error free communication. So for every request signal from the controller placed at the tank, an acknowledgement signal is transmitted from the controller at the sump. Suppose an error or loss of data occurs during transmission, then the system is programmed to re-transmit the data.

\section{PIC MICROCONTROLLER}

PIC microcontrollers (Programmable Interface Controllers) are electronic circuits that can be programmed to carry out a vast range of tasks. The PIC is a very general purpose microcontroller that can come with many different options, for very reasonable prices. PIC16F877A is the largest chip of the 16F87x family; has 8 analogue inputs, this is probably the most popular PIC used in engineering community that is still under production. This is the best PIC of its family for bigger engineering projects, along with the PIC16F84 for smaller ones. Its major Features includes: $14 \mathrm{~KB}$ of program memory, 368 bytes of RAM, a 40 pin package, 8 ADC channels capable of 10-bit each [11].

\subsection{Ask Based RF Module}

The radio frequency (RF) transmission system in this project employs Amplitude Shift Keying (ASK) with transmitter/receiver $(\mathrm{Tx} / \mathrm{Rx})$ pair operating at $433 \mathrm{MHz}$ License Free Band. The transmitter module takes serial input and transmits these signals through $\mathrm{RF}$. The transmitter signals are received by the receiver module placed away from the source of transmission [12].

\subsubsection{ASK Transmitter module (Tx)}

The system allows one way communication between two nodes, namely, transmission and reception. The RF module has been used in conjunction with a set of four channel encoder/decoder ICs. The HT12E \& HT12D have been used as encoder and decoder respectively. The encoder converts the parallel inputs (from the tank probes) into serial set of signals. These signals are serially transferred through $\mathrm{RF}$ to the reception point. The decoder is used after the RF receiver has received the signal to decode the serial format and retrieve the original signals as outputs. 


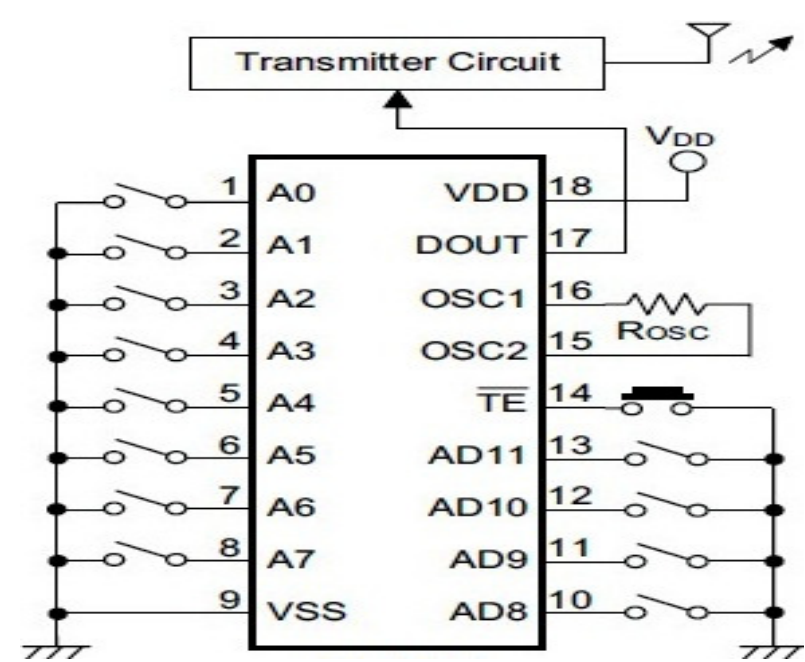

Figure 3: Encoder/RF transmitter sub-circuit

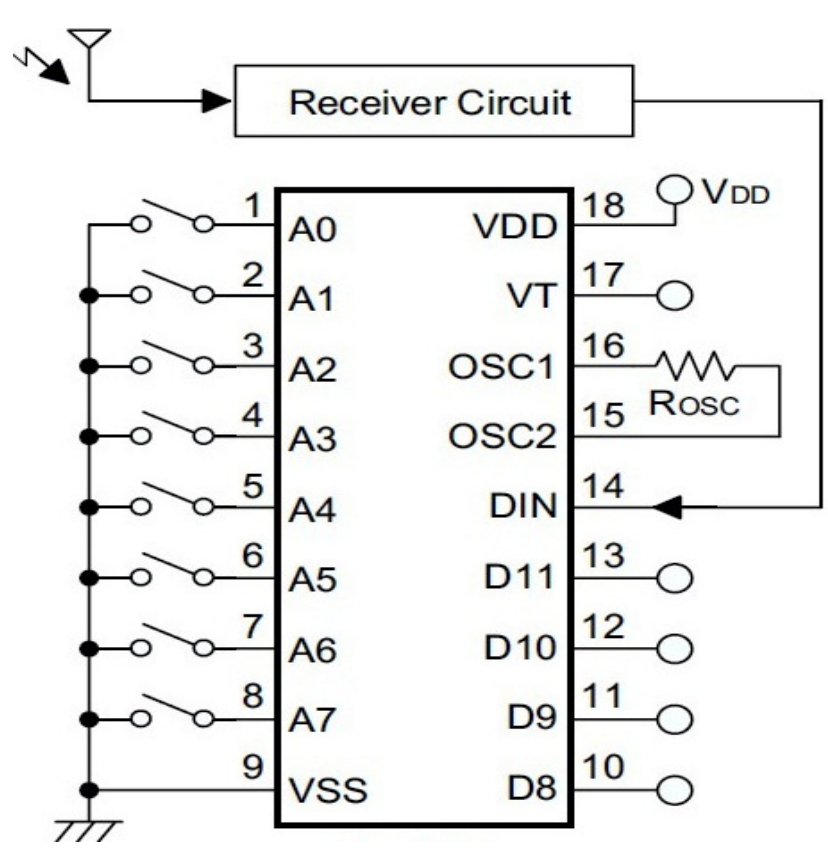

Figure 4: Receiver/decoder sub-circuit

\subsubsection{Encoder/Transmitter Sub-Circuit:}

This circuit has the task of sensing, encoding and transmitting the water level of the prototype miniature overhead tank to the receiver/controller part. The HT12E Encoder IC shown in Figure 3 will convert the 4 bit parallel data given to pins D0 - D3 to serial data and will be available at DOUT. This output serial data is given to ASK (Amplitude Shift Keying) RF Transmitter. Address inputs A0 - A7 can be used to provide data security and can be connected to GND (Logic ZERO) or left open (Logic ONE).

\subsection{Decoder/Receiver Sub-Circuit}

This circuit has the task of receiving and decoding the data sent from the encoder/transmitter circuit mounted on the prototype miniature overhead tank at $433 \mathrm{MHz}$ frequency.
ASK (Amplitude Shift Keying) RF Receiver receives the data transmitted using ASK RF Transmitter. HT12D decoder of Figure 4 will convert the received serial data to 4 bit parallel data D0 - D3. The status of these address pins A0-A7 should match with status of address pin in the HT12E at the transmitter for the transmission of data. The LED connected to the above circuit glows when valid data transmission occurs from transmitter to receiver. $51 \mathrm{~K} \Omega$ resistor will provide the necessary resistance required for the internal oscillator of the HT12D [14]. The circuit is powered from the +5 voltage output of the power supply unit which can be seen from the Figure 4 above.

\subsection{Basic Control Logic:}

This is the basic control logical interpretation needed for this particular work, and is responsible for the sensing, display; buzzing and water pump control in this system using a 4-bit code for each level sensing.

\begin{tabular}{cccccc}
\multicolumn{7}{c}{ Table 2: 4-bit control logic } \\
\hline Water & 4-bit & Hex & LCD & Buzzer & Water \\
Level & Code & Equivalent & Display & State & Pump \\
\hline Full & 0000 & 00 & $100 \%$ & 2 secs & Off \\
High & 1000 & 08 & $75 \%$ & Off & NO \\
Mid & 1100 & OC & $50 \%$ & Off & NO \\
Low & 1110 & OE & $25 \%$ & Off & On \\
very low & 1111 & OF & below $25 \%$ & 2 secs & On \\
\hline \multicolumn{7}{c}{ NO - no operation }
\end{tabular}

From the above logic table, when the water level in the tank is sensed by the sensor assembly, which is connected to the 4-bit data lines (AD8 through AD11) of encoder HT12E. Depending on the water level in the tank, the 4-bit code so generated is encoded by the encoder HT12E. The encoded data is fed to pin 2 of RF transmitter (TX1), which transmits it serially at 433 MHz through the antenna connected to its (pin 1).

The 4-bit signal from the transmitter is received by the antenna of the RF receiver (RX1). LED1 glows to indicate that a valid signal is received. The 4-bit output from decoder HT12D is processed by the microcontroller to generate an 8-bit code. The microcontroller's output is fed to data input lines of LCD, and indicates the water level in percentage.

At an intermediate water level of (1110), 25\% of the tank capacity, LCD shows the message "Water level $25 \%$ ". When the tank is full at (0000), the buzzer rings for two seconds, while LCD shows "Water level 100\%". The pump operation can also be observed from the above table, the pump is activated at logic (1110) $25 \%$ and (1111) below 25\%, the microcontroller shorts down the pump at logic (0000) 100\%, and displays the 
pump state on the same LCD. The logic (1111) below $25 \%$ arises only in the time of fault, i.e. if the pump fails to switch on when it is activated via the controller, and then the owner will be notified of the fault by the buzzer for two seconds.

\subsubsection{Microcontroller Programming.}

The coding process starts by defining the available input data with respect to the PIC16F877A. The table below details the input pins, and the data to the microcontroller in relation to the sensor encoder pins. Basic logic conversions

$$
\begin{aligned}
& 0000_{2}=00_{16} \\
& 1000_{2}=08_{16} \\
& 1100_{2}=0 \mathrm{C}_{16} \\
& 1110_{2}=0 \mathrm{E}_{16} \\
& 1111_{2}=0 \mathrm{~F}_{16}
\end{aligned}
$$

\section{Table.3: Microcontroller 4-bit input data}

\begin{tabular}{cccccc}
\hline \multicolumn{3}{c}{$\begin{array}{c}\text { Microcontroller 4-bit data } \\
\text { input pins }\end{array}$} & $\begin{array}{c}\text { Hex } \\
\text { Equivalent }\end{array}$ & $\begin{array}{c}\text { Encoder } \\
\text { Pins }\end{array}$ \\
\hline$A O$ & $A 1$ & $A 2$ & $A 3$ & & $D O-D 4$ \\
0 & 0 & 0 & 0 & 0x00 & D4 \\
1 & 0 & 0 & 0 & 0x08 & D3 \\
1 & 1 & 0 & 0 & 0x0C & D2 \\
1 & 1 & 1 & 0 & 0x0E & D1 \\
1 & 1 & 1 & 1 & 0x0F & D0 \\
\hline
\end{tabular}

The PIC16F877A has 40 pins made up of 5 pots (A, B, C, $D$, and E). For this work only ports (A, B, C and D) are needed: Port " $A$ " is the input (data from decoder) while Port " $\mathrm{B}$ " is the output (data to LCD). Also, Port " $\mathrm{C}$ " is the output (data to LEDs) and Port "D" becomes the output (control of pump and buzzer)

For the programming ISIS Proteus 8 pro simulation software and Micro C pro assembler for PIC were used. MikroC PRO for PIC is a full-featured C language based compiler for PIC devices from Microchip ${ }^{\circledR}$. This software is used to burn the program in to PIC microcontroller since it has powerful compiler with advanced optimizations, lots of hardware and software libraries, and additional tools that will helped in this your work. The flow chart of Figure 5 is used to program the microcontroller.

\subsection{Power Supply Unit:}

A constant low voltage regulated power supply of $+5 \mathrm{~V}$, as is shown in Figure 7, provides input voltages to the PIC16F877A, LCD module, and LEDs which requires 5 volts supply and $+12 \mathrm{~V}$, providing input voltage to the relay sub-circuit is needed. To ensure that the system performs reliably economically, it is better to power the control circuit with mains input power, but the main challenge with mains supply voltage is of its alternating form and the magnitude available at 220 volts is too high for the components making up the control circuit.

\subsubsection{Relay Sub-Circuit:}

Relays were used in building a switching unit that simply triggers the pump on or off, depending on the signal received from the microcontroller. The process of powering the relay takes place in Figure 6 above, when the transistor is biased by a "high" signal from the PIC16F877A the relay will remain energized as long as the signal from the PIC microcontroller pin RD1 remains high resulting in water pump in "active mode". When the pump control mode changes, the PIC microcontroller signal output to the transistor goes "low" resulting in no AC power for the $0.5 \mathrm{hp}$ water pump to run "Off line mode".

From Figure 7, diode (D1) is in parallel with the relay coil. The diode serves as a clamp or fly-back mechanism for the relay.

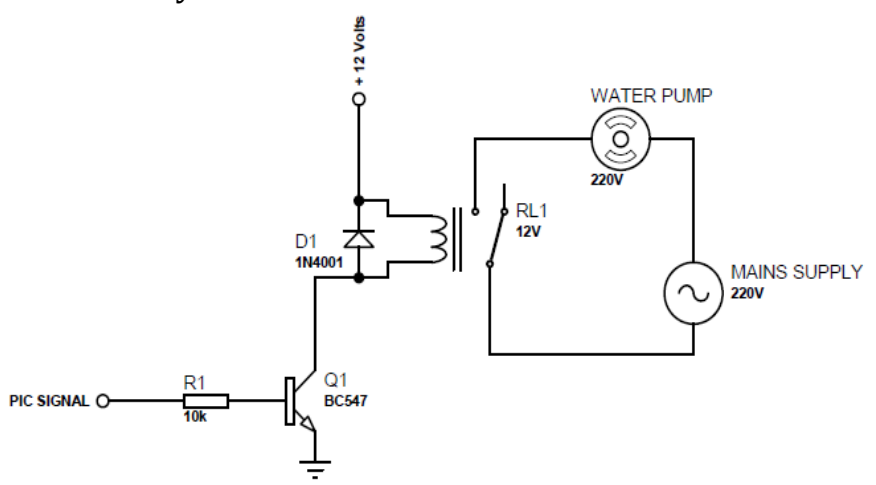

Figure 7: Relay Sub-Circuit

\subsubsection{Relay circuit calculations}

$$
I_{B}=\frac{I_{c}}{\beta}
$$

$I_{C}$ is the collector current and $\beta$ is the given as the gain of the transistor, given as 150 (from datasheet).

$I_{C}=\mathrm{V} / \mathrm{R}$

$\mathrm{V}$ is the applied voltage ( $\mathrm{V}=12$ volts), $\mathrm{R}$ is the resistance offered by the relay internal circuitry. ( $R=$ $400 \Omega)$.

$I_{C}=12 \mathrm{~V} / 400 \Omega=30 \mathrm{~mA}$

Hence, $I_{B}=30 / 105 \mathrm{~mA}=0.2 \mathrm{~mA}$

For effective biasing of the transistor, it is customary to make $I_{B}$ value to be twice the obtained value.

Therefore the value of the base current used is: $2 * 0.2 \mathrm{~mA}=0.4 \mathrm{~mA}$. 


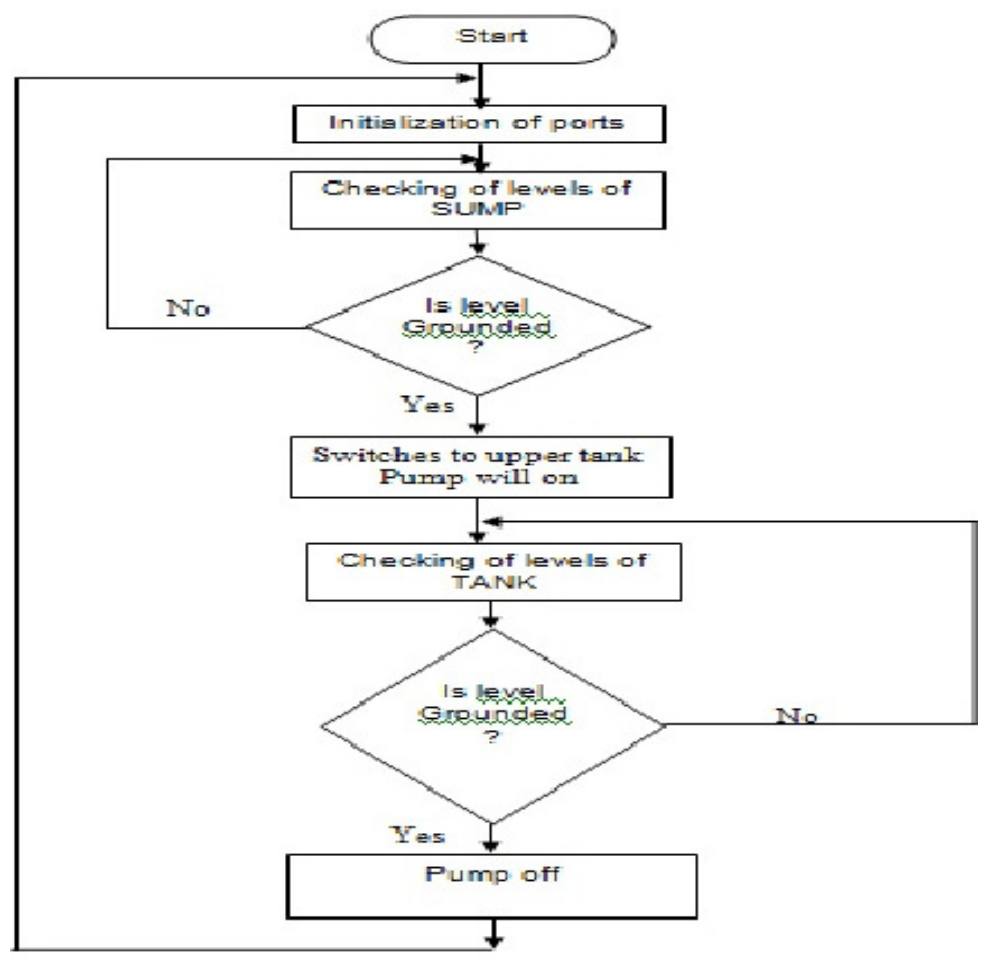

Figure 5 Flow chart of system design

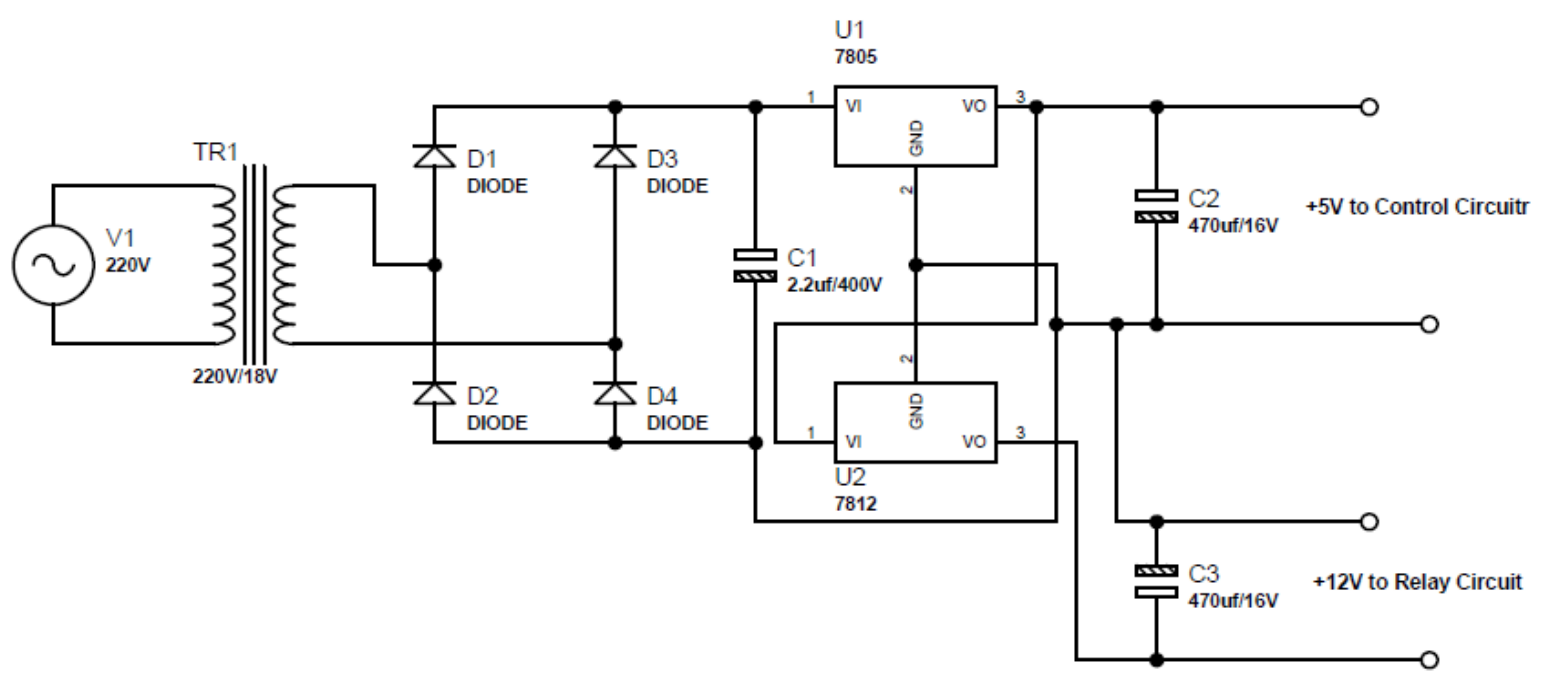

Fig.6: Power Supply Unit Circuit

The PIC microcontroller that serves as the current source to the transistor has an output voltage:

$V_{m}=4.95$ Volts

$V_{m}=V_{m} / I_{B}$

Therefore, $R_{B}=4.95 / 0.4 \mathrm{~mA}=12,375 \approx 12 \mathrm{k} \Omega$.

Since $12 \mathrm{k} \Omega$ resistor doesn't exist, a $10 \mathrm{k} \Omega$ resistor is applied for this work. $R_{B}$ Chosen $=10 \mathrm{k} \Omega$.

\subsection{Control System Sub-Circuit:}

The brain of the controlling section for this work is the 40 pin PIC16F877A microcontroller. It processes the data received from the Receiver Section. LCD Display, LED indications and Motor status are updated according to the data the PIC receives at a particular time. For the transmission and reception of data the HOLTEK encoder-decoder pair of HT12E and HT12D is used. Both of them are CMOS (Complementary metaloxide-semiconductor) family of ICs with working voltage ranges from 2.4 to 12 volts see Figure 8 below. The oscillator resistances are chosen according to the datasheet. When water level rises, the data pins of the encoder will be grounded corresponding to the level of water, which will be transmitted to the Receiver via ASK, RF module. The received data is Decoded by the decoder HT12D. 


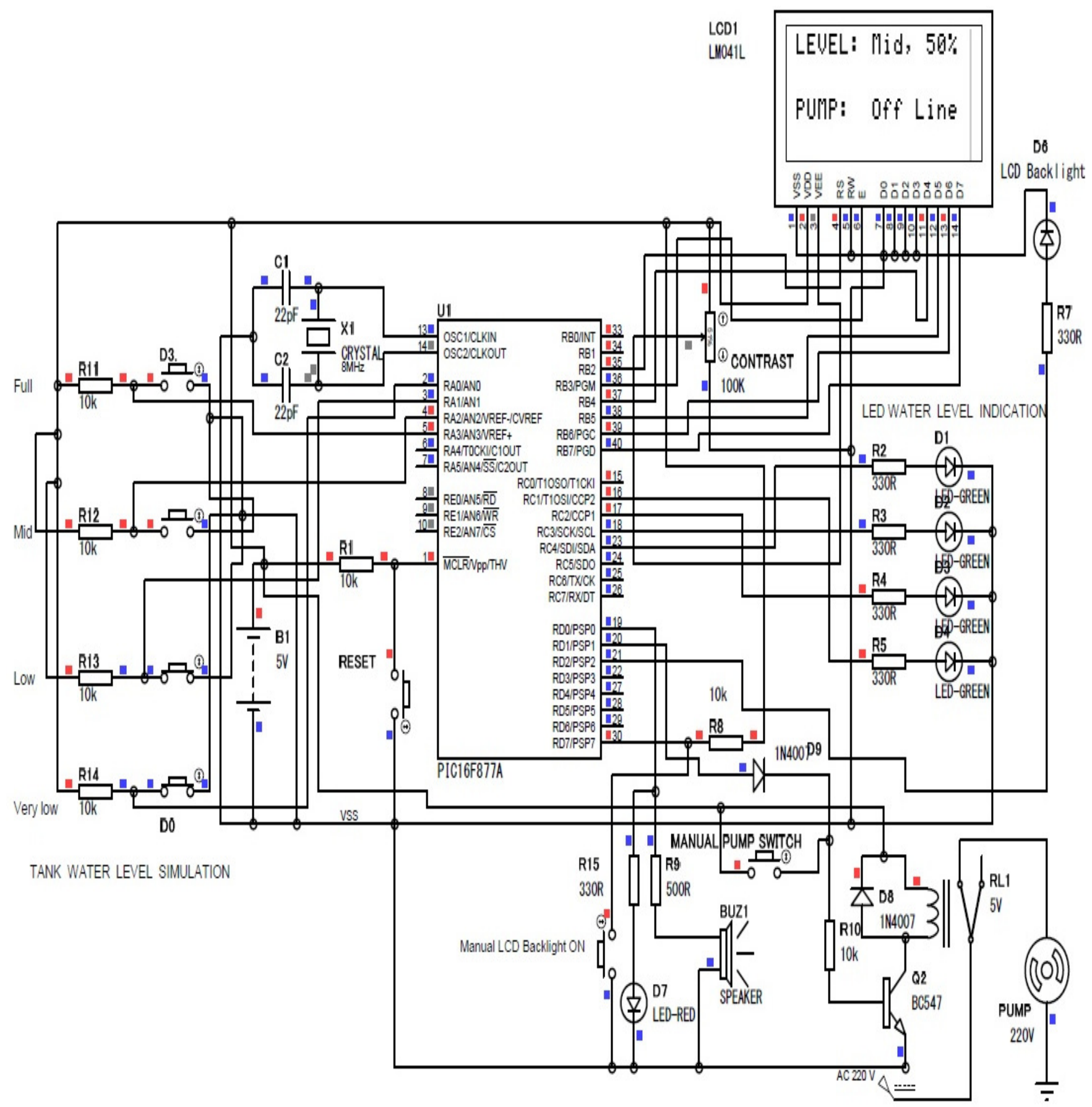

Figure 8: Control system sub-circuit

The LED on the receiver indicates that it is receiving data. Then the data is given to the PIC for processing. When the water level becomes very low, the motor will turned ON, buzzer sounds and the LCD backlight will automatically turned $\mathrm{ON}$ for 5 seconds. After this, when the water level reaches full level, the motor will automatically turned OFF, buzzer sounds and the LCD backlight will automatically turned $\mathrm{ON}$ for 5 seconds. During normal operation the LCD backlight can be manually turn on by pressing the Push button switch. The LCD indicates the Level of water ('Very Low', 'Low', 'Medium', 'High', 'Full') and the status of the motor
('Activated' or 'Off line') is displayed simultaneously on the LCD. The LED bar will also indicate the water level and was included as a backup for the LCD. From Figure 8 resistor (R11 to R14) are pull-up resistors of $10 \mathrm{k} \Omega$ each, they set the input pins of the PIC pin (A0 to A3) at a "high". Resistor R1 of $10 \mathrm{k} \Omega$ is configured with the reset button for effective PIC rest when needed. While Resistors (R2 to R5) are current limiting resistors for the green indicator LEDs (D1 to D4) and Resistor R7 of $330 \Omega$ limits the current to the LCD backlight appropriately. 


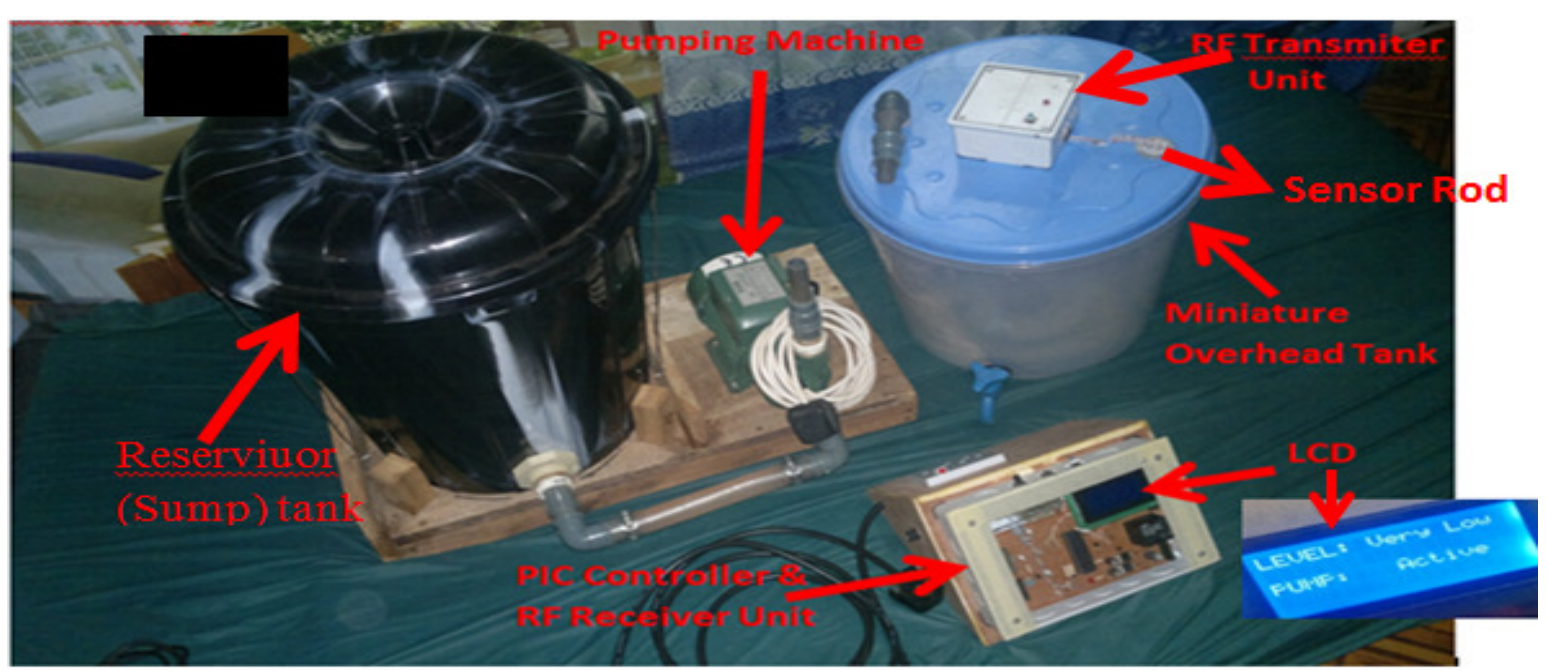

Figure 9: Complete RF water level indicator controller project assembly

\subsection{Sensor Unit}

Water level sensor unit consists of two parts, one sensor is used in reservoir tank (sump) and other four sensors placed inside the overhead tank. Moreover, sensors are composed with rod, nozzles, inducting rubber etc. Rod is made by iron and steel, that is connected with ground. Nozzles are connected with $+5 \mathrm{v}$. Copper rod and nozzles are bound together via a rubber. Rubber is used to make the electrical connection of copper rod and nozzles separate. Due to water conductivity $33 \mathrm{k} \Omega$ resistance has been used. The basic operation is, when one nozzle of the sensor is drawn into water, nozzle and rod becomes connected due to water conductivity. Then nozzle gets ground signal $(0 \mathrm{v})$ which is connected with input of the microcontroller which makes LEDs on/off, acts as user display unit.

Table 4: Technical specifications

\begin{tabular}{llc}
\hline \multirow{2}{*}{ Voltage } & Operating Voltage & 150V to 240V AC \\
& Sensing Voltage & $-5 \mathrm{~V} D \mathrm{CC}$ \\
& Control Voltage & $+5 \mathrm{~V} \mathrm{DC}$ \\
\hline \multirow{2}{*}{ Current } & Sensor Current & $5.5 \mathrm{~mA}$ \\
& Controller Current & $250 \mathrm{~mA}$ \\
\hline \multirow{2}{*}{ Power } & Sensor Power & $0.0275 \mathrm{~W}$ \\
& Controller Power & $1.25 \mathrm{~W}$ \\
\hline \multirow{2}{*}{ Frequency } & Operating Frequency & $50 \mathrm{~Hz}$ \\
& Controller Frequency & $8 \mathrm{MHz}$ \\
& Module Frequency & $433 \mathrm{MHz}$ \\
\hline \multirow{2}{*}{ Temperature } & \multirow{2}{*}{ Operating Temperature } & $25{ }^{0} \mathrm{C} \mathrm{to} 50{ }^{\circ} \mathrm{C}$ \\
\hline
\end{tabular}

\subsection{Performance Test}

This test involved synchronizing whole system, from the tank/pipe leakage test to the receiver/controller and sensor/transmitter test, the project was at this point subjected to rigorous and stressful testing so as to ensure its durability and stability. Up to 100 meters distance RF data transmission from tank to controller was achieved during this test.

\section{CONCLUSION:}

The aim of this work was to design and implement a prototype of a microcontroller-radio frequency based water level indicator / controller system which automatically sense accurately the water level of an overhead tank and automatically activate or deactivate a single phase water pump for industrial and domestic applications. The control unit can power conveniently up to $2 \mathrm{hp}$ pump. Using this system, one can remotely monitor and control the water level of an overhead tank that is placed up to 100 meters away. The level of water in the overhead tank is being monitored and at certain level of water $25 \%$ empty, the system automatically switches the pumping machine on to pump water in to the overhead tank and then switches off when the water is $100 \%$ full. The intention of this research work is to establish a flexible, economical and easy configurable system which can solve the problem of water wastage.

The system has been tested and found to meet all the expected results. After multiple tests carried out with this prototype, one can confidently say that the work is stable, efficient, durable, and easy to maintain. The prototype is shown in Figure 9.

\section{REFERENCE}

[1] The Deeter Group Sensors, Float Switches \& Level Sensors from Deeter Electronics Inc. http://www.deeterelectronicsinc.com/products/flo at-switch-liquid-level-sensors- overview Retrieved on 7-11- 2014. 
[2] Al-Naamany, A. M.; Meribout, M.; and Al Busaidi, K., "Design and Implementation of a New Nonradioactive-Based Machine for Detecting OilWater Interfaces in Oil Tanks", IEEE Transactions on Instrumentation and Measurement, Volume 56, Issue 5, pp. $1532-1536$, Oct. 2007.

[3] Mahmoud Meribout, Ahmed Al Naamany and Khamis Al Busaidi, "Interface Layers Detection in Oil Field Tanks: A Critical Review, Expert Systems for Human, Materials and Automation", Prof. PetricÄfVizureanu (Ed.), ISBN: 978-953-307-334-7, In Tech, http://www.intechopen.com/books/expertsystemsfor-human-materials-and automation/interface-layers-detection-in-oil-fieldtanks-a-critical-review pp. 181 -208, 2011.

[4] G.A. Kalinov, A.V. Lysakov, V.I. Rimlyand, "HighPrecision Ultrasonic Water Level Indicator" XXII Session of the Russian Acoustical Society Moscow, Session of the Scientific Council of Russian Academy of Science on Acoustics, pp254 - 256, June 15-17, 2010.

[5] Jonathan D. Weiss, "DA \& Control A Novel Fiber-Optic Fluid Interface Sensor", Sensor online Technical Resource for sensing communication and control, May 1, 2008.

[6] K. Govinda; D. Anupreetham; C. SriSaiVivek, "Online Reservoir Water Level Indicator - Design and Applications", IJSRD - International Journal for Scientific Research \& Development| Vol. 2, Issue 03, pp.599 - 601, 2014

[7] Khaled Reza, S.M., Shah Ahsanuzzaman Md. Tariq, S.M. Mohsin Reza, "Microcontroller Based Automated Water Level Sensing and Controlling: Design and
Implementation Issue", Proceedings of the World Congress on Engineering and Computer Science, pp 220- 224, 2010.

[8] M. Javanmard, K.A. Abbas and F. Arvin, "A Microcontroller-Based Monitoring System for Batch Tea Dryer", CCSE Journal of Agricultural Science, Vol. 1, No. 2, December 2009

[9] Ejiofor Virginia Ebere and Oladipo Onaolapo Francisca, "Microcontroller based Automatic Water level Control System", International Journal of Innovative Research in Computer and Communication Engineering, Vol. 1, Issue 6, pp. 1390 - 1396, August 2013.

[10] Texas Instrument CC2500Low-Cost Low-Power2.4 GHzRF Transceiver data book, Copyright(C2014, Texas Instruments Incorporated.

[11] Microchip PIC16F882/883/884/886/887 Data Sheet, 28/40/44-Pin, Enhanced Flash-Based 8-Bit CMOS Microcontrollers with NanoWatt Technology, 2009, Microchip Technology Incorporated.

[12] RWS-371 RF MODULE Series Wireless Hi Sensitivity Receiver Module (RF ASK) data sheet, http://www.wenshing.com.tw;http://www.rf.net.tw Retrieved on 7-11-2014.

[13] HOLTEK $2^{12}$ Series of Encoders datasheet, 15th Mar '96, www.eleinmec.com/datasheets/ ds holtek ht12d.pd Retrieved on 7-11-2014.

[14] HOLTEK $2^{12}$ Series of Decoders datasheet, 15th Mar '96, fromwww.eleinmec.com/datasheets/ds_holtek_ht12 d.pdRetrieved on 7-11-2014 